\title{
Asymptotic Formulas for the Reflection/Transmission of Long Water Waves Propagating in a Tapered and Slender Harbor
}

\author{
Eric-Gustavo Bautista, ${ }^{1}$ Federico Méndez, $^{2}$ and Oscar Bautista ${ }^{1}$ \\ ${ }^{1}$ Instituto Politécnico Nacional, SEPI ESIME Azcapotzalco, Avenida de las Granjas No. 682, Colonia Santa Catarina, \\ Delegación Azcapotzalco, 02250 México, DF, Mexico \\ ${ }^{2}$ Departamento de Termofluidos, Facultad de Ingeniería, UNAM, 04510 México, DF, Mexico
}

Correspondence should be addressed to Eric-Gustavo Bautista; erbautista@ipn.mx

Received 29 April 2015; Accepted 7 July 2015

Academic Editor: Michael Meylan

Copyright (C) 2015 Eric-Gustavo Bautista et al. This is an open access article distributed under the Creative Commons Attribution License, which permits unrestricted use, distribution, and reproduction in any medium, provided the original work is properly cited.

We obtain asymptotic formulas for the reflection/transmission coefficients of linear long water waves, propagating in a harbor composed of a tapered and slender region connected to uniform inlet and outlet regions. The region with variable character obeys a power-law. The governing equations are presented in dimensionless form. The reflection/transmission coefficients are obtained for the limit of the parameter $\kappa_{2} \ll 1$, which corresponds to a wavelength shorter than the characteristic horizontal length of the harbor. The asymptotic formulas consider those cases when the geometry of the harbor can be variable in width and depth: linear or parabolic among other transitions or a combination of these geometries. For harbors with nonlinear transitions, the parabolic geometry is less reflective than the other cases. The reflection coefficient for linear transitions just presents an oscillatory behavior. We can infer that the deducted formulas provide as first approximation a practical reference to the analysis of wave reflection/transmission in harbors.

\section{Introduction}

Long water waves can propagate over large distances from the coast line along slender harbors. In some cases, the geometry of these structures can be strongly tapered, producing the amplification of the wave amplitude; see Li et al. [1]. A property of oscillations in harbors is that even relatively small vertical motions can be accompanied by large horizontal motions of the water, Wiegel [2]; under these circumstances, the oscillations could create unacceptable vessel motions and excessive mooring forces leading to breaking of mooring lines as it was detailed by Rabinovich [3].

Inside the harbors can be presented different configurations and connections between two or more basins through a narrow passage $[4,5]$; at least one of these is open to the sea, and it is identified as outer harbor and the others are identified as inner harbors. The outer harbor can act like a filter and reduces the energy of waves propagating towards the inner harbor; see, for instance, Bellotti [6].
When the water wave is travelling from the outer harbor entrance, it is strongly affected by the geometry of the harbor. In harbors, a small portion of the water waves are reflected repeatedly by the interior boundaries. Some portions of the wave energy escape to the ocean, while others stay inside the harbor, Mei et al. [7]. The wave reflected is an important mechanism that may endanger harbor access, due to sea states at the entrance, Zanuttigh et al. [8]. In addition, if the harbor geometry converges in a narrow passage, we can appreciate the motion of waves with large amplitudes. The reflection and the amplification of the water waves by tapered and slender harbors are effects that should be taken into account in their design and are characterized by the reflection and transmission coefficients.

Different researchers conducted fundamental studies related to the propagation of water waves in complex geometries with the aid of experimental and theoretical models [9, 10], made specially to obtain the reflection and transmission coefficients. Among others, one important case that has 
been widely studied is the propagation of water waves over an infinite step [11, 12] and the deformation of long water waves onto a shelf with abrupt and gradual changes in the depth; see, for example, Goring [13]. On the other hand, the linear long water wave approximation has been used to estimate the reflection and transmission coefficients for different submerged trapezoidal breakwaters, Chang and Liou [14]. In this direction, Jung et al. [15] obtained an approximated analytical solution for the mild-slope equation, applied to the analysis of wave reflection for an asymmetric trench. The aforementioned work was improved by Xie and Liu [16] by obtaining an exact analytical solution to the modified mild-slope equation. Bender and Dean [17] studied the reflection and transmission of normally incident waves by two-dimensional trenches and shoals. Wang et al. [18] using the linear shallow water approximation analyzed the longitudinal oscillations in a rectangular harbor with a hyperbolic-cosine squared bottom induced by incident perpendicular waves. Lin and Liu [19] obtained an analytical solution for linear long wave reflection by an obstacle of general trapezoidal shape. In the same direction, Xie et al. [20] explored the linear long wave reflection by a rectangular obstacle with two scour trenches of power function profile and later Liu et al. [21] proposed an exact analytical solution in the form of a Taylor series for linear long wave reflection by a submerged quasi-idealized breakwater or trench. In addition, there are studies applying perturbation techniques to predict the reflection and modulation of long water waves propagating over geometries with variable depth and in channels with constant depth and parabolic width transition; see, for example, [22, 23].

In this work, we obtain asymptotic formulas for the reflection/transmission coefficients of linear long water waves, propagating in a harbor composed of a tapered and slender region connected to uniform inlet and outlet regions. The coefficients are derived in the limit for which the wavelength is smaller than the characteristic horizontal length of a harbor. In order to obtain the above formulas, we have adopted the analytical solution for the free surface elevation of linear long water waves propagating in a tapered and slender channel proposed by Bautista et al. [24], which is obtained with the WKB singular perturbation technique. We consider obviously those cases where the geometry of the harbor can be variable in width and depth: linear or parabolic among other transitions or a combination of these geometries. To verify the derived formula for the reflection coefficient, it is compared against the classical formula for the reflection of linear long water waves propagating on a depth with linear transition. The influence of the dimensions and shape of the harbors on the reflection and transmission coefficients is investigated. The easy application of the reflection/transmission coefficients allows us to clarify which geometrical transitions of the harbor are less or more reflective.

\section{Formulation}

Let us consider a linear long water wave propagating from right to left hand side in a tapered and slender harbor. We

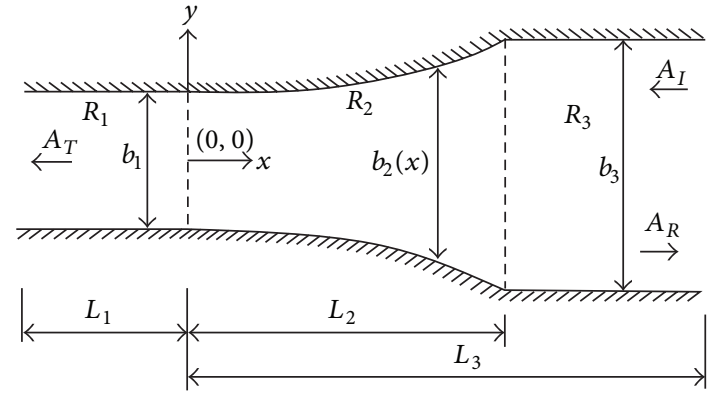

FIgURE 1: The top view diagram of the physical model studied. The water wave amplitude $A_{w}$ for $w=I, R$, and $T$ represents the incident (travelling from right to the left hand side), reflected, and transmitted water waves, respectively. The harbor is formed for three regions $R_{1}, R_{2}$, and $R_{3}$.

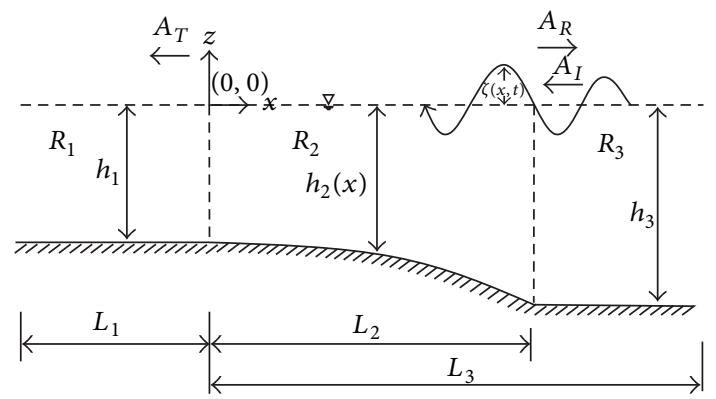

FIgURE 2: The side view diagram of the physical model studied. The water wave amplitude $A_{w}$ for $w=I, R$, and $T$ represents the incident (travelling from right to the left hand side), reflected, and transmitted water waves, respectively. The harbor is formed for three regions $R_{1}, R_{2}$, and $R_{3}$.

assume that the harbor is formed by three regions $R_{1}, R_{2}$, and $R_{3}$, where region $R_{2}$ defines just the variable character of the cross section, while regions $R_{1}$ and $R_{3}$ assume uniform values for the width and depth of the harbor; see Figures 1 and 2 . In the selected Cartesian coordinate system, the $x$-axis is positive to the right with origin placed in the intersection of regions $R_{1}$ and $R_{2}, y$-axis is pointing out in the transversal direction of the harbor, and the $z$-axis is pointing outwards in a normal direction to the mean sea water level. The interval where the transition region $R_{2}$ occurs is $0 \leq x \leq L_{2}$, while the regions $R_{1}$ and $R_{3}$ are defined by the intervals $-L_{1} \leq$ $x \leq 0$ and $L_{2} \leq x \leq L_{3}$, respectively, where $L_{1}$ and $L_{2}$ are the lengths of the regions $R_{1}$ and $R_{2}$, respectively, while $L_{3}$ corresponds to a length measure from the origin of the reference system $x=0$ to the inlet of the region $R_{3}$. In the present analysis, we assume that the walls of the harbor are impermeable. However, the vertical boundaries on the right and left sides of the system are completely open to the flow of the water waves. In particular, we assume the sum of incident and reflected waves on the right and only a transmitted wave 
on the left. The prescribed widths and depths of the harbors are given by the following relationships:

$$
\begin{array}{r}
b(x)= \begin{cases}b_{1} & \text { for }-L_{1} \leq x \leq 0 \\
b_{2}(x)=\beta x^{m}+b_{1} & \text { for } 0 \leq x \leq L_{2} \\
b_{3} & \text { for } L_{2} \leq x \leq L_{3}\end{cases} \\
h(x)= \begin{cases}h_{1} & \text { for }-L_{1} \leq x \leq 0 \\
h_{2}(x)=\alpha x^{n}+h_{1} & \text { for } 0 \leq x \leq L_{2} \\
h_{3} & \text { for } L_{2} \leq x \leq L_{3} .\end{cases}
\end{array}
$$

In the above equations, the slenderness ratios $\alpha$ and $\beta$ are defined by

$$
\begin{aligned}
& \beta=\frac{b_{3}-b_{1}}{L_{2}^{m}}, \\
& \alpha=\frac{h_{3}-h_{1}}{L_{2}^{n}} .
\end{aligned}
$$

In addition, the geometry shapes of the width and the depth of the harbors are given by the values of $m \geq 1$ and $n \geq 1$, respectively, with $m, n \sim O(1)$. The arguments, for which $m$ and $n$ can not be less than one, are explained in Section 3. The power-law functions for the width and the depth, given by (1), are taken as geometrical representations or approximations to tapered and slender harbors.

Under some specific circumstances, in harbors, the water depth is very much smaller compared with the wavelength. Therefore, the wave oscillations can be approximated as shallow water waves, for which water flows are mostly in the horizontal planes, while the vertical velocity variations are weak and the pressure is hydrostatic as a first approximation. The fluid flow is governed by the unidimensional linear continuity and momentum equations, Mei et al. [7, p. 124]:

$$
\begin{array}{r}
\frac{\partial \zeta(x, t)}{\partial t}+\frac{1}{b(x)} \frac{\partial[U(x, t) h(x) b(x)]}{\partial x}=0, \\
\frac{\partial U(x, t)}{\partial t}+g \frac{\partial \zeta(x, t)}{\partial x}=0,
\end{array}
$$

where $\zeta(x, t)$ is the water surface elevation and $U(x, t)$ is the mean water velocity in the $x$ direction; for simplicity, hereafter $h=h(x), t$ is the time and $g$ is the gravitational acceleration.

The continuity and momentum equations (3) and (4) can be combined in only one. By this purpose, we can derive (3) with respect to the time and replace (4) in the resulting equation, obtaining the following equation:

$$
\frac{\partial^{2} \zeta(x, t)}{\partial t^{2}}-\frac{g}{b(x)} \frac{\partial}{\partial x}\left[h(x) b(x) \frac{\partial \zeta(x, t)}{\partial x}\right] .
$$

For infinite regions of the wave propagation, typically the wavelength (but not necessarily) is selected as the characteristic horizontal length, Svendsen [25, p. 328]; however, for the present work, which has a well defined geometry, the horizontal characteristic scale over which changes the magnitude of the dependent variables is the length $L_{2}$, the characteristic time scale of the wave is the period $T$, and the characteristic scale for the wave amplitude is the incident wave amplitude $A_{I}$. For nondispersive linear long water waves the characteristic wave celerity is given by $c=\sqrt{g h_{1}}=$ $\omega / k$, where the wave frequency is $\omega=2 \pi / T$, the wave number is given by $k=2 \pi / \lambda$, and the wavelength in region $R_{1}$ is denoted by $\lambda$.

Therefore, for region $R_{2}$, we can introduce the following dimensionless variables:

$$
\begin{aligned}
\chi_{2} & =\frac{x}{L_{2}}, \\
\Delta_{2} & =\frac{\zeta(x, t)}{A_{I}}, \\
\tau & =\omega t .
\end{aligned}
$$

Substituting the previous dimensionless variables into (5), the dimensionless governing equation for linear long waves propagating in a tapered and slender harbor $\left(b_{3} / L_{2} \ll 1\right)$ is given by

$$
\frac{\partial^{2} \Delta_{2}}{\partial \tau^{2}}-\frac{\kappa_{2}}{\bar{b}\left(\chi_{2}\right)} \frac{\partial}{\partial \chi_{2}}\left[G\left(\chi_{2}\right) \frac{\partial \Delta_{2}}{\partial \chi_{2}}\right]=0
$$

where $G\left(\chi_{2}\right)=\bar{b}\left(\chi_{2}\right) \bar{h}\left(\chi_{2}\right)$ is the dimensionless area of the transversal section of the harbor in the transition region $R_{2}$, the dimensionless width is written as $\bar{b}\left(\chi_{2}\right)=\left(1-\varepsilon_{w}\right) \chi_{2}^{m}+$ $\varepsilon_{w}$, and the dimensionless depth is expressed as $\bar{h}\left(\chi_{2}\right)=(1-$ $\left.\varepsilon_{h}\right) \chi_{2}^{n}+\varepsilon_{h} ; \varepsilon_{h}=h_{1} / h_{3}$ and $\varepsilon_{w}=b_{1} / b_{3}$. In the above equation, the dimensionless parameter $\kappa_{2}$ is defined as

$$
\kappa_{2}=\left(\frac{\lambda}{2 \pi L_{2}}\right)^{2}=\frac{g h_{3}}{\left(\omega L_{2}\right)^{2}} .
$$

The parameter $\kappa_{2}=\left[\lambda /\left(2 \pi L_{2}\right)\right]^{2}$ represents the competition between the wavelength, $\lambda$, and the characteristic horizontal length of the harbor, $L_{2}$. From a physical point of view, the limit of $\kappa_{2} \ll 1$ corresponds to the horizontal projection $L_{2}$, very large compared with $\lambda$.

Along the three regions of the harbor, we assume that the waves are harmonic functions in time and can be expressed as

$$
\Delta_{j}\left(\chi_{j}, \tau\right)=\operatorname{Re}\left[\delta_{j}\left(\chi_{j}\right) e^{-i \tau}\right],
$$

where $j=1,2$, and 3 indicates the regions $R_{1}, R_{2}$, and $R_{3}$, respectively. The dimensionless amplitudes of the water waves at any cross section of the harbor are denoted by $\delta_{j}\left(\chi_{j}\right), i=$ $\sqrt{-1}$ and Re "is the real part of."

For the region $R_{2}$ and from (9), we suggest the following initial conditions:

$$
\begin{aligned}
\Delta_{2}\left(\chi_{2}, 0\right) & =\operatorname{Re}\left[\delta_{2}\left(\chi_{2}\right)\right], \\
\frac{\partial \Delta_{2}\left(\chi_{2}, 0\right)}{\partial \tau} & =\operatorname{Re}\left[-i \delta_{2}\left(\chi_{2}\right)\right] .
\end{aligned}
$$


It should be noted that, in (10), the unknown function $\delta_{2}\left(\chi_{2}\right)$ must be determined as a part of the problem.

In addition, (7) requires appropriated boundary conditions for $\Delta_{2}\left(\chi_{2}, \tau\right)$ at $\chi_{2}=0$ and $\chi_{2}=1$. These conditions are obtained from the continuity condition for the total head pressure at the junctions $[7,26]$.

We define the following dimensionless variables:

$$
\begin{aligned}
\chi_{1} & =-\frac{x}{L_{1}}, \\
\chi_{3} & =\frac{x-L_{2}}{L_{3}-L_{2}}, \\
\bar{Z} & =\frac{z}{h_{3}} \\
\bar{P}_{j}\left(\chi_{j}, \bar{Z}, \tau\right) & =\frac{P_{j}(x, z, t)}{\rho g h_{3}}, \\
\Delta_{1}\left(\chi_{1}, \tau\right) & =\frac{\zeta_{1}(x, t)}{A_{I}}, \\
\Delta_{3}\left(\chi_{3}, \tau\right) & =\frac{\zeta_{3}(x, t)}{A_{I}},
\end{aligned}
$$

where $j=1,2$, and 3 indicates the regions $R_{1}, R_{2}$, and $R_{3}$, respectively, $P_{j}(x, z, t)$ is the pressure, $\rho$ is the water density, and $\zeta_{1}(x, t)$ and $\zeta_{3}(x, t)$ are the free surface elevations in the regions $R_{1}$ and $R_{3}$, respectively.

The pressure under linear long water waves is still hydrostatic; that is, $P_{j}(x, z, t)=\rho g\left[\zeta_{j}(x, t)-z\right]$, Dean and Dalrymple [27, p. 133]. Substituting the dimensionless variables (11) into the previous relationship, we obtain the dimensionless version of the pressure

$$
\bar{P}_{j}\left(\chi_{j}, \bar{Z}, \tau\right)=\varepsilon \Delta_{j}\left(\chi_{j}, \tau\right)-\bar{Z}
$$

where $\varepsilon=A_{I} / h_{3}$.

Considering that the pressures at the connection between the regions $R_{1}-R_{2}$ and $R_{2}-R_{3}$ are equals, we have that

$$
\begin{aligned}
& \bar{P}_{2}\left(\chi_{2}=0, \bar{Z}, \tau\right)=\bar{P}_{1}\left(\chi_{1}=0, \bar{Z}, \tau\right), \\
& \bar{P}_{2}\left(\chi_{2}=1, \bar{Z}, \tau\right)=\bar{P}_{3}\left(\chi_{3}=0, \bar{Z}, \tau\right) .
\end{aligned}
$$

Substitution of (12) into (13) and (14) leads to

$$
\begin{aligned}
& \Delta_{2}\left(\chi_{2}=0, \tau\right)=\Delta_{1}\left(\chi_{1}=0, \tau\right), \\
& \Delta_{2}\left(\chi_{2}=1, \tau\right)=\Delta_{3}\left(\chi_{3}=0, \tau\right) .
\end{aligned}
$$

Introducing (9) into (7) and (15), the following boundary value problem is obtained:

$$
\begin{aligned}
& \kappa_{2} \frac{d^{2} \delta_{2}\left(\chi_{2}\right)}{d \chi_{2}^{2}}+\frac{\kappa_{2}}{G\left(\chi_{2}\right)} \frac{d G\left(\chi_{2}\right)}{d \chi_{2}} \frac{d \delta_{2}\left(\chi_{2}\right)}{d \chi_{2}}+\frac{\delta_{2}\left(\chi_{2}\right)}{\bar{h}\left(\chi_{2}\right)} \\
& \quad=0
\end{aligned}
$$

with the following boundary conditions:

$$
\begin{aligned}
& \delta_{2}\left(\chi_{2}=0\right)=\delta_{1}\left(\chi_{1}=0\right), \\
& \delta_{2}\left(\chi_{2}=1\right)=\delta_{3}\left(\chi_{3}=0\right) .
\end{aligned}
$$

In the above boundary conditions $(17), \delta_{1}\left(\chi_{1}=0\right)$ and $\delta_{3}\left(\chi_{3}=0\right)$ are the unknown dimensionless amplitudes of the linear long water waves propagating in regions $R_{1}$ and $R_{3}$, respectively, which are presented in paragraphs below.

The asymptotic solution of (16) for the limit of $\kappa_{2} \ll 1$, obtained with the WKB perturbation technique and reported in detail by Bautista et al. [24], is adopted for the present work and it is given by

$$
\delta_{2}\left(\chi_{2}\right)=\frac{1}{\bar{h}^{1 / 4}\left(\chi_{2}\right) \bar{b}^{1 / 2}\left(\chi_{2}\right)}\left(C e^{i \vartheta_{x}}+D e^{-i \vartheta_{x}}\right) .
$$

Equation (18) is derived for a channel composed of a region with variables width and depth and connected to inlet and outlet regions which extend with infinite lengths, conversely to the equivalent regions analyzed in the present work, where we assume well defined lengths; however, the infinite lengths have no influence on the behavior of the waves in the region of the harbor with variable section, because the patching boundary conditions are not functions of the lengths of the regions with constant width and depth. Therefore, the above analytical solution is maintained valid for the present work.

In (18), the constants $C$ and $D$ must be determined from the appropriated patching boundary conditions. On the other hand, $\vartheta_{x}=F\left(\chi_{2}, n\right) / \kappa_{2}^{1 / 2}$ and $F\left(\chi_{2}, n\right)$ is defined as

$$
F\left(\chi_{2}, n\right)=\int \frac{1}{\sqrt{\bar{h}\left(\chi_{2}\right)}} d \chi_{2} .
$$

Following a similar procedure as that developed for the region $R_{2}$ and taking into account relationships (11) and (9), the dimensionless surface elevation for regions $R_{1}$ and $R_{3}$ can be determined by solving the following differential equation:

$$
\frac{d^{2} \delta_{j}\left(\chi_{j}\right)}{d \chi_{j}^{2}}+\kappa_{j}^{2} \delta_{j}\left(\chi_{j}\right)=0,
$$

where $j=1$ and 3 indicates the regions $R_{1}$ and $R_{3}$, respectively. The analytical solutions of (20) for the water waves amplitude in regions $R_{1}$ and $R_{3}$ are trivial and are given by

$$
\begin{aligned}
& \delta_{1}\left(\chi_{1}\right)=\beta_{T} e^{i \kappa_{1} \chi_{1}}, \\
& \delta_{3}\left(\chi_{3}\right)=\beta_{R} e^{i \kappa_{3} \chi_{3}}+e^{-i \kappa_{3} \chi_{3}},
\end{aligned}
$$

with $\kappa_{1}=\left(\omega L_{1}\right) /\left(g h_{1}\right)^{1 / 2}, \kappa_{3}=\omega\left(L_{3}-L_{2}\right) /\left(g h_{3}\right)^{1 / 2}, \beta_{R}=$ $A_{R} / A_{I}$, and $\beta_{T}=A_{T} / A_{I}$.

In the above equations, $A_{R}$ and $A_{T}$ are the unknown complex amplitudes of the reflected and transmitted waves, respectively. Evaluating (21) at $\chi_{1}=0$, we can obtain that $\delta_{1}\left(\chi_{1}=0\right)=\beta_{T}$ and, for $\chi_{3}=0, \delta_{3}\left(\chi_{3}=0\right)=\beta_{R}+1$. These relationships are just the unknown boundary conditions in (17). 


\section{Reflection and Transmission Coefficients for the Limit of $\kappa_{2} \ll 1$}

To obtain the values of the coefficients $C, D, \beta_{R}$, and $\beta_{T}$, we require two additional boundary conditions, which can be obtained from the continuity of mass flux at the connections between the regions $R_{1}-R_{2}$ at $\chi_{2}=0$ and $R_{2}-R_{3}$ at $\chi_{2}=1$. Taking into account the above comments the corresponding patching boundary conditions are given by the following dimensionless relationships:

$$
\begin{aligned}
& \left.\frac{d \delta_{1}}{d \chi_{1}}\right|_{\chi_{1}=0}=-\left.\Phi \frac{d \delta_{2}}{d \chi_{2}}\right|_{\chi_{2}=0}, \\
& \left.\frac{d \delta_{2}}{d \chi_{2}}\right|_{\chi_{2}=1}=\left.\widetilde{\Phi} \frac{d \delta_{3}}{d \chi_{3}}\right|_{\chi_{3}=0},
\end{aligned}
$$

where $\Phi=L_{1} / L_{2}$ and $\widetilde{\Phi}=L_{2} /\left(L_{3}-L_{2}\right)$.

From (17), (18), and (23), we can easily derive the following system of equations:

$$
\begin{aligned}
& {\left[\begin{array}{cccc}
e^{i \vartheta_{1}} & e^{-i \vartheta_{1}} & -1 & 0 \\
{\left[1+a_{2} i\right] e^{i \vartheta_{1}}} & -\left[1-a_{2} i\right] e^{-i \vartheta_{1}} & -1 & 0 \\
e^{i \vartheta_{2}} & e^{-i \vartheta_{2}} & 0 & -a_{0} \\
{\left[1+a_{0} a_{1} \varepsilon_{h}^{1 / 2} i\right] e^{i \vartheta_{2}}} & -\left[1-a_{0} a_{1} \varepsilon_{h}^{1 / 2} i\right] e^{-i \vartheta_{2}} & 0 & a_{0}
\end{array}\right]\left[\begin{array}{c}
C \\
D \\
\beta_{R} \\
\beta_{T}
\end{array}\right]} \\
& =\left[\begin{array}{c}
1 \\
-1 \\
0 \\
0
\end{array}\right]
\end{aligned}
$$

with

$$
\begin{aligned}
\vartheta_{1}= & \frac{F\left(\chi_{2}=1, n\right)}{\kappa_{2}^{1 / 2}}, \\
\vartheta_{2}= & \frac{F\left(\chi_{2}=0, n\right)}{\kappa_{2}^{1 / 2}}, \\
a_{0}= & \varepsilon_{h}^{1 / 4} \varepsilon_{w}^{1 / 2}, \\
a_{1}= & \alpha_{1} \kappa_{2}^{1 / 2}, \\
a_{2}= & \alpha_{2} \kappa_{2}^{1 / 2}, \\
\alpha_{1}= & \frac{m\left(1-\varepsilon_{w}\right) q\left(\chi_{2}=0, m\right)}{2 a_{0} \varepsilon_{w}^{1 / 4}} \\
& +\frac{n\left(1-\varepsilon_{h}\right) g\left(\chi_{2}=0, n\right)}{4 a_{0} \varepsilon_{h}}, \\
\alpha_{2}= & \frac{m\left(1-\varepsilon_{w}\right)}{2}+\frac{n\left(1-\varepsilon_{h}\right)}{4} .
\end{aligned}
$$

In the above set of equations, $F\left(\chi_{2}=0, n\right)$ and $F\left(\chi_{2}=\right.$ $1, n)$ are the solutions of integral (19), evaluated at $\chi_{2}=$ 0 and $\chi_{2}=1$, respectively, for different values of the exponent $n$ with $n \geq 1$. In addition, in relationships (25) for $\alpha_{1}, g\left(\chi_{2}, n\right)=\chi_{2}^{n-1}$, and $q\left(\chi_{2}, m\right)=\chi_{2}^{m-1}$, these functions for values of $m, n>1$ and $m, n \sim O(1)$ must be evaluated at $\chi_{2}=0$ obtaining that $g\left(\chi_{2}=0, n\right)=0$ and $q\left(\chi_{2}=0, m\right)=0$ and, for harbors with linear transition, $(m=1$ and/or $n=1)$, are reduced to $g\left(\chi_{2}=0, n\right)=q\left(\chi_{2}=0, m\right)=1$. It is very important to note that the exponents $n$ and $m$ can take different values, depending on the harbor shape geometry, that is, $(1,1.5,2,2.5,3,5)$. In order to satisfy the condition of $\lambda \ll L_{2}$, represented by $\kappa_{2} \ll 1$ and slender harbor, given by $b_{3} / L_{2} \ll 1$, the values of $m$ and $n$ must be restricted to be of order unity. Harbors with $m, n \gg 1$ degenerate in an abrupt transition as $\chi_{2} \rightarrow 1$, and therefore the slender assumption is no longer satisfied. It is possible to combine different shapes of the width and the depth. In this mathematical model, the values of $m$ and $n$ must be greater than or equal to unity, because the power-law distribution has the origin located at $\chi_{2}=0$, just in the intersection of regions $R_{1}$ and $R_{2}$ and, for the case of $n<1$ and $m<1$, the functions $g\left(\chi_{2}, n\right)$ and $q\left(\chi_{2}, m\right)$ are divergent and, therefore, (18) will be also undefined.

The solution of the system of (24) is trivial and is given by

$$
\begin{aligned}
C & =-i \frac{2 a_{1} a_{0}^{2} \varepsilon_{h}^{1 / 2}}{\Omega} e^{i \vartheta_{1}}, \\
D & =\frac{2 a_{0}\left(2+a_{1} a_{0} \varepsilon_{h}^{1 / 2} i\right)}{\Omega} e^{i\left(\vartheta_{1}+2 \vartheta_{2}\right)}, \\
\beta_{R} & =\frac{1}{\Omega}\left[a_{0}^{2} a_{1} \varepsilon_{h}^{1 / 2}\left(a_{2}-2 i\right) e^{i 2 \vartheta_{1}}\right. \\
& \left.-a_{0} a_{2}\left(a_{0} a_{1} \varepsilon_{h}^{1 / 2}-2 i\right) e^{i 2 \vartheta_{2}}\right],
\end{aligned}
$$

$$
\beta_{T}=\frac{4}{\Omega} e^{i\left(\vartheta_{1}+\vartheta_{2}\right)}
$$

with $\Omega=a_{0}\left(a_{2}+2 i\right)\left(a_{0} a_{1} \varepsilon_{h}^{1 / 2}-2 i\right) e^{i 2 \vartheta_{2}}-a_{0}^{2} a_{1} a_{2} \varepsilon_{h}^{1 / 2} e^{i 2 \vartheta_{1}}$.

To obtain the reflection and transmission coefficients, we make an energy flow balance: at $\chi_{2}=0$ only a transmitted wave is presented, while at $\chi_{2}=1$ there are incident and reflected waves; therefore, in a simplified form, the energy flux balance is given by

$$
C_{R}^{2}+\varepsilon_{w} \varepsilon_{h}^{1 / 2} C_{T}^{2}=1
$$

In the above equation, the reflection and transmission coefficients $C_{R}=\left|\beta_{R}\right|$ and $C_{T}=\left|\beta_{T}\right|$ can be written as

$$
\begin{aligned}
C_{R} & =\mid \frac{1}{\Omega}\left[a_{0}^{2} a_{1} \varepsilon_{h}^{1 / 2}\left(a_{2}-2 i\right) e^{i 2 \vartheta_{1}}\right. \\
& \left.-a_{0} a_{2}\left(a_{0} a_{1} \varepsilon_{h}^{1 / 2}-2 i\right) e^{i 2 \vartheta_{2}}\right] \mid, \\
C_{T} & =\frac{4}{|\Omega|}
\end{aligned}
$$

where | | represents the module of a complex number. 
Taking into account relationships (25), for harbors with width transition $m>1$ and $\varepsilon_{h}=1,(28)$ and (29) can be written in a simplified form as

$$
\begin{aligned}
& C_{R} \approx \frac{m\left(1-\varepsilon_{w}\right) \kappa_{2}^{1 / 2}}{4}, \\
& C_{T} \approx \frac{1}{\varepsilon_{w}^{1 / 2} \sqrt{1+\alpha_{2}^{2} \kappa_{2} / 4}}
\end{aligned}
$$

respectively. In a similar form as before, for harbors with depth transition $n>1$ and $\varepsilon_{w}=1,(28)$ and (29) are reduced to

$$
\begin{aligned}
& C_{R} \approx \frac{n\left(1-\varepsilon_{h}\right) \kappa_{2}^{1 / 2}}{8}, \\
& C_{T} \approx \frac{1}{\varepsilon_{h}^{1 / 4} \sqrt{1+\alpha_{2}^{2} \kappa_{2} / 4}}
\end{aligned}
$$

respectively.

Equations (28)-(33) are developed for slow variations of the depth and width of the harbor, $\left(h_{3} / L_{2} \ll 1\right.$ and $b_{3} / L_{2} \ll$ 1 ), and for a characteristic length of the harbor bigger than the wavelength $\left(\kappa_{2} \ll 1\right)$, and for sufficiently small amplitude $\left(A_{I} / h_{3} \ll 1\right)$.

For the limit of $\varepsilon_{w} \rightarrow 1$ and $\varepsilon_{h} \rightarrow 1$, the coefficients take values of $C_{R} \approx 0$ and $C_{T} \approx 1$.

Now, from the dimensionless generalized Green's law, Lighthill [28, p. 122],

$$
\delta_{2}\left(\chi_{2}=0\right) \sim \frac{1}{\left[\bar{h}^{1 / 4}\left(\chi_{2}=0\right) \bar{b}^{1 / 2}\left(\chi_{2}=0\right)\right]},
$$

and, from the definition of the transmission coefficient $C_{T}=$ $\left|\beta_{T}\right| \sim O\left[\delta_{2}\left(\chi_{2}=0\right)\right]$, we have that

$$
C_{T} \sim \frac{1}{\left[\bar{h}^{1 / 4}\left(\chi_{2}=0\right) \bar{b}^{-1 / 2}\left(\chi_{2}=0\right)\right]}
$$

with $\bar{h}\left(\chi_{2}=0\right)=\varepsilon_{h}$ and $\bar{b}\left(\chi_{2}=0\right)=\varepsilon_{w}$; therefore, for the limits of $\varepsilon_{h} \ll 1$ and $\varepsilon_{w} \ll 1$, the second term on the left side of $(27)$ is $\left(\varepsilon_{w} \varepsilon_{h}^{1 / 2} C_{T}^{2}\right) \rightarrow 1$, which implies that, to preserve the energy flow, for the above limits, the reflection coefficient must be $C_{R} \ll 1$, as is shown in Results and Discussion.

From the above comments, in this work, for the limits of $\varepsilon_{w} \ll 1$ and $\varepsilon_{h} \ll 1$, the reflection coefficient can not be approximated to one and the transmission coefficient can not be approximated to zero, because the present mathematical model is derived for tapered and slender harbors, as was previously commented on. The geometrical conditions for which $C_{R}$ could be approximated to one are those cases of rapidly varying cross section or abrupt transitions, geometrical conditions that are out of the scope of the present work and are not treated here.

Equations (28) and (29) allow obtaining values of the reflection and transmission coefficients for harbors with

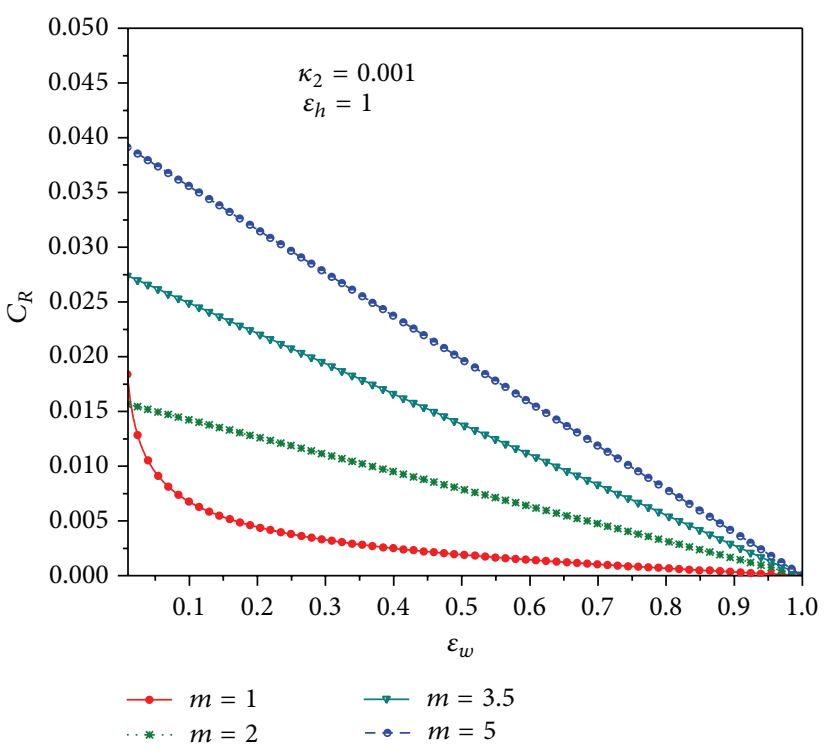

FIGURE 3: Reflection coefficient as a function of the dimensionless parameter $\varepsilon_{w}$, for waves propagating in a harbor with width transitions $m(=1,2,3.5,5)$, with $\varepsilon_{h}=1$ and $\kappa_{2}=0.001$.

linear or parabolic transitions among other transitions or a combination of these geometries.

For the limit of $\kappa_{2} \ll 1,(28)$ and (29) can reproduce the classical case of linear long wave reflection by a depth with linear transition reported by Dean [29], as can be verified in Section 4.2 of Results and Discussion. Although the values of the coefficient $C_{R}$, obtained for the limit of $\kappa_{2} \ll 1$ and calculated from (28), are small, these allow to identifying those geometrical transitions that are less or more reflective.

\section{Results and Discussion}

In order to investigate the influence of the harbor geometry on the reflection and transmission of linear long water waves, as an example, we use the following typical physical data: the length and width of the harbor are $L_{2}=3500 \mathrm{~m}$ and $b_{3}=300 \mathrm{~m}$, respectively, and a depth $h_{3}=25 \mathrm{~m}$, Zwamborn and Grieve [30]; however, because the present mathematical formulation is expressed in dimensionless form, many other representative values of the physical variables can be used. In addition, we propose two widths $b_{1}(=300 \mathrm{~m}$ and $75 \mathrm{~m})$ and two depths $h_{1}(=25 \mathrm{~m}$ and $6.25 \mathrm{~m})$; with these values we obtain the width and depth ratios $\varepsilon_{w}(=1,0.25)$ and $\varepsilon_{h}(=1,0.25)$, respectively. In order to satisfy the long water wave theory, the wavelength in region $R_{3}$ is $\lambda=695.4 \mathrm{~m}$; therefore, the kinematical parameter takes a value of $\kappa_{2}=$ 0.001. In this manner, the selected data satisfy the assumption of long water wave theory, which in this case is equivalent to $h_{3} / \lambda=0.036 \leq 1 / 20$; see Rahman [31, p. 161] and the fundamental assumption is fulfilled and satisfied.

4.1. Wave Reflection by a Harbor with Constant Depth and Variable Width. In Figure 3, we show the results of the reflection coefficient $C_{R}$ as a function of the width ratio $\varepsilon_{w}$ 


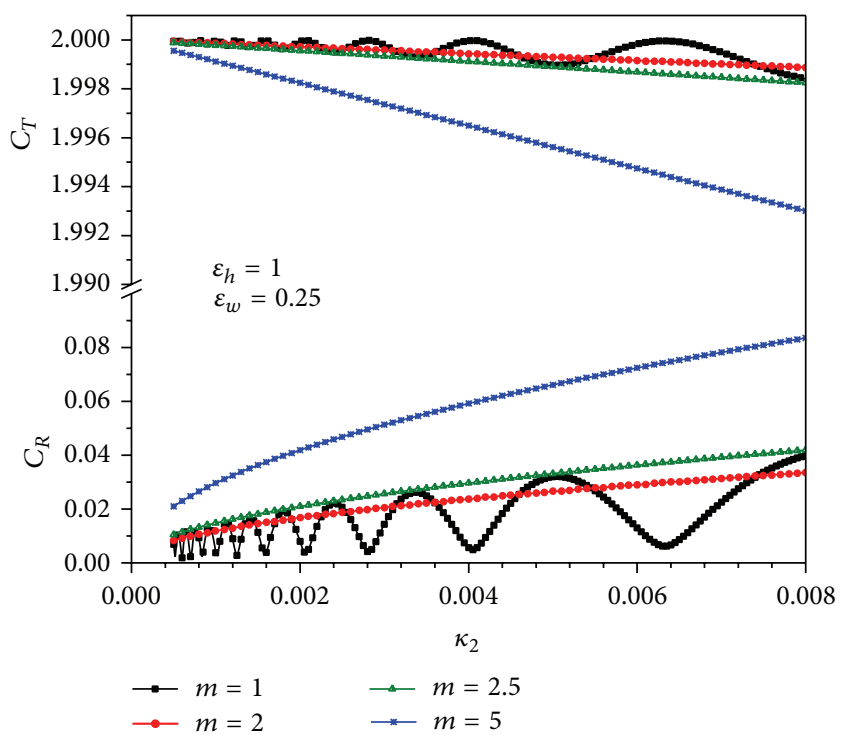

FIGURE 4: Reflection/transmission coefficients as a function of the kinematical parameter $\kappa_{2}$ with $\varepsilon_{h}=1$ and $\varepsilon_{w}=0.25$ for four values of $m(=1,2,2.5,5)$.

for different values of $m(=1,2,3.5,5), \varepsilon_{h}=1$, and $\kappa_{2}=0.001$. The results of the coefficient $C_{R}$ for a harbor with a linear transition width $(m=1)$ are obtained by using $(28)$ and for those cases of harbors with $m>1$ and $\varepsilon_{h}=1$, we use (30). From (30) it can be deduced that the reflection coefficient for a specific geometry of the width transition $m$ increases or decreases its magnitude depending on the assumed values of the parameter $\kappa_{2}=\left[\lambda /\left(2 \pi L_{2}\right)\right]^{2}$. For values of the parameter $\kappa_{2}$ such that $L_{2} \rightarrow \lambda$, the harbors are more reflective than the case with $L_{2} \gg \lambda$. On the other hand, for constant values of the parameters $\kappa_{2}$ and $\varepsilon_{w}$, the reflection coefficient increases or decreases its magnitude proportionally in terms of the values of $m$, where always $m>1$ and $m \sim O(1)$.

In general, from Figure 3 we show that a harbor with linear width transition is less reflective than harbors with nonlinear transitions; however, as $\varepsilon_{w} \rightarrow 0$, the reflection coefficient for $m(=2,3.5,5)$ takes finite values of $C_{R}(=$ $0.01581,0.02766,0.03949)$, respectively. While the harbor with $m=1$, the reflection coefficient increases its values exponentially.

In Figure 4, the behaviors of the reflection $C_{R}$ and transmission $C_{T}$ coefficients as a function of the parameter $\kappa_{2}$ are plotted for four values of $m(=1,2,2.5,5)$ with constant values of $\varepsilon_{w}=0.25$ and $\varepsilon_{h}=1$. For the cases of $m>1$ the results of reflection coefficients are calculated from (30) and for $m=1$ we use (28). The transmission coefficients $C_{T}$ for the values of $m>1$ and $m=1$ are obtained from (31) and (29), respectively.

From (31) with $\kappa_{2} \rightarrow 0$, which means that $L_{2} \gg$ $\lambda$, the transmission coefficient is $C_{T} \sim O\left(\varepsilon_{w}^{-1 / 2}\right)$ and the harbor geometry has no influence on the wave transmission; however, for values of $\kappa_{2}$ such that $L_{2} \rightarrow \lambda$ the term $\alpha_{2}^{2} \kappa_{2} / 4$ must be retained in order to identify the influence of harbor geometry on the wave transmission.

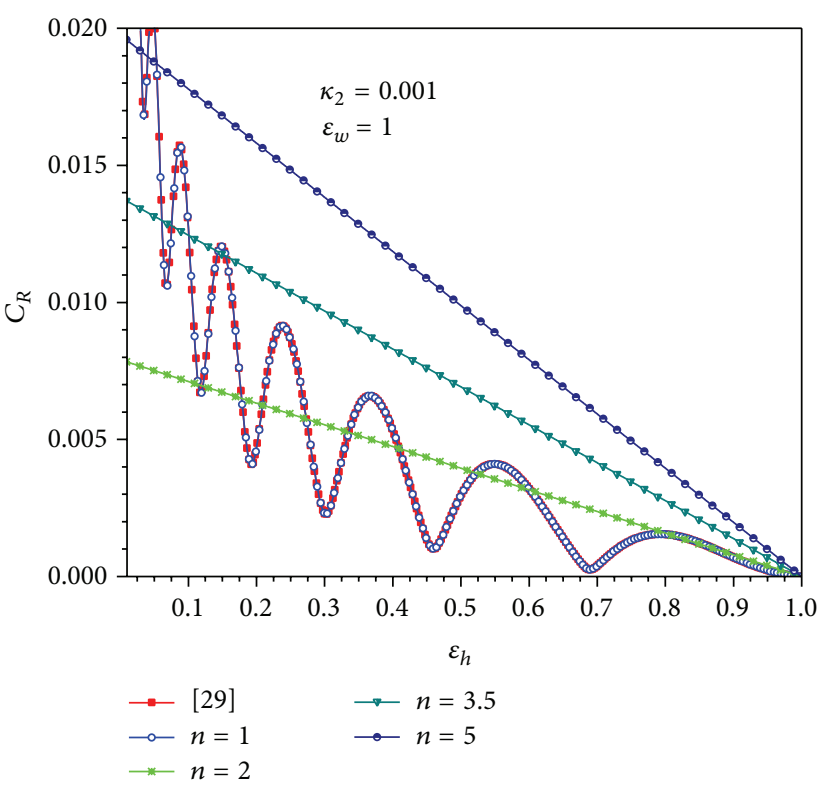

FIGURE 5: Reflection coefficient as a function of the dimensionless parameter $\varepsilon_{h}$, for waves propagating on a depth with transitions $n(=$ $1,2,3.5,5)$ with $\varepsilon_{w}=1$ and $\kappa_{2}=0.001$.

In general, Figure 4 reveals that for large values of the parameter $\kappa_{2}$ the coefficient $C_{R}$ increases and $\mathrm{C}_{T}$ decreases. From a physical point of view, this condition is presented only for $L_{2} \rightarrow \lambda$; otherwise, for $L_{2} \gg \lambda$ the harbors are less reflective. In the limit of $C_{R} \rightarrow 0,(27)$ is simplified to $C_{T}=$ $\varepsilon_{h}^{-1 / 4} \varepsilon_{w}^{-1 / 2}$; therefore, we can substitute the values of $\varepsilon_{w}=0.25$ and $\varepsilon_{h}=1$ to obtain that $C_{T}=2$, which is the maximum value observed in Figure 4. Under these conditions, the maximum value of the transmission coefficient for this case is $C_{T} \sim$ $O\left(\varepsilon_{w}^{-1 / 2}\right)$. It can be appreciated that, in the interval plotted for the parameter $\kappa_{2}$ and with the exception of $m=1$, the other transition shapes do not have an oscillatory behavior, this characteristic also can be observed in (30) and (31), which do not include oscillating terms, and these are only presented in the generalized formulas used to obtain the coefficients $C_{R}$ and $C_{T}$ for the harbor with linear transition width, (28) and (29), respectively.

4.2. Wave Reflection by a Harbor with Constant Width and Variable Depth. In Figure 5, we show the values of the reflection coefficient $C_{R}$ as a function of the depth ratio $\varepsilon_{h}$ with constant values of $\kappa_{2}=0.001$ and $\varepsilon_{w}=1$ and four values of $n(=1,2,3.5,5)$. From (32), we obtain similar results to those obtained in Section 4.1, for specific values of the parameters $n, \varepsilon_{h}$, and $\kappa_{2}$. For constant values of the parameters $\kappa_{2}$ and $\varepsilon_{h}$ the reflection coefficient increases its magnitude for large values of $n>1$ and $n \sim O(1)$. Comparing the nonlinear transitions, just the shape geometry with $n=2$ is less reflective than those cases of $n(=3.5$ and 5); see Figure 5 .

For the particular case of $n=1$, the reflection coefficient is calculated from (28) and it is compared against the analytical 


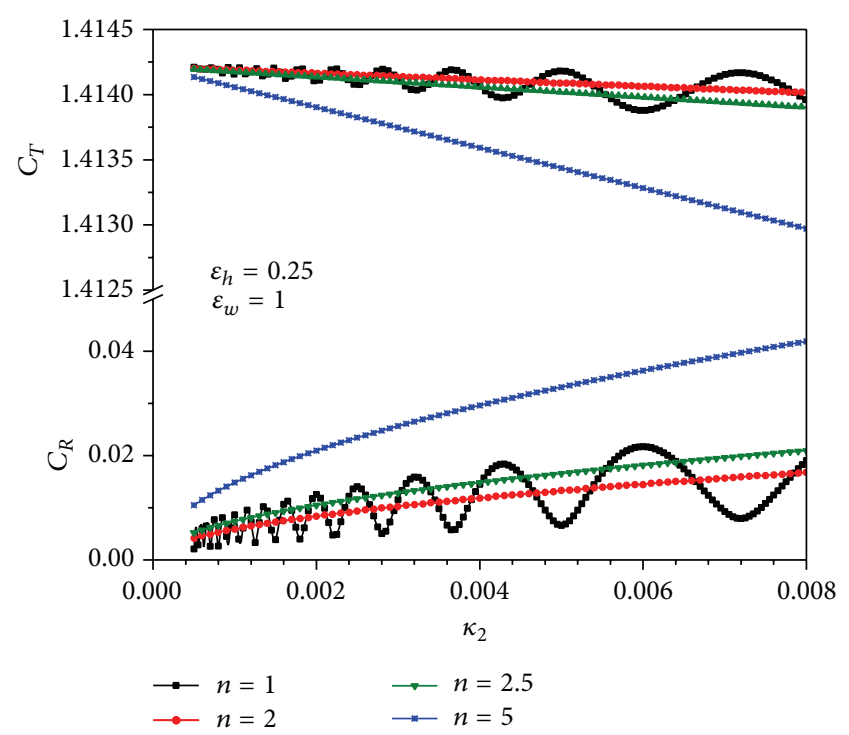

FIGURE 6: Reflection/transmission coefficients as a function of the kinematical parameter $\kappa_{2}$ with $\varepsilon_{w}=1$ and $\varepsilon_{h}=0.25$ for four values of $n(=1,2,2.5,5)$.

model proposed by Dean [29], which is given by

$$
C_{R}=\frac{\sqrt{\gamma_{0} M_{1}+\gamma_{1} M_{0}-2 v_{0} v_{1}-8 / \pi^{2} E_{0}^{2} \varepsilon_{h}^{1 / 2}}}{\sqrt{\gamma_{0} M_{1}+\gamma_{1} M_{0}-2 v_{0} v_{1}+8 / \pi^{2} E_{0}^{2} \varepsilon_{h}^{1 / 2}}},
$$

where

$$
\begin{aligned}
E_{0} & =4 \pi\left(\frac{L_{2}}{\lambda}\right) \frac{1}{1-\varepsilon_{h}}, \\
E_{1} & =4 \pi\left(\frac{L_{2}}{\lambda}\right) \frac{\varepsilon_{h}^{1 / 2}}{1-\varepsilon_{h}}, \\
\gamma_{0} & =J_{0}^{2}\left(E_{0}\right)+J_{1}^{2}\left(E_{0}\right), \\
\gamma_{1} & =J_{0}^{2}\left(E_{1}\right)+J_{1}^{2}\left(E_{1}\right), \\
M_{0} & =Y_{0}^{2}\left(E_{0}\right)+Y_{1}^{2}\left(E_{0}\right), \\
M_{1} & =Y_{0}^{2}\left(E_{1}\right)+Y_{1}^{2}\left(E_{1}\right), \\
v_{0} & =J_{0}\left(E_{0}\right) Y_{0}\left(E_{0}\right)+J_{1}\left(E_{0}\right) Y_{1}\left(E_{0}\right), \\
\nu_{1} & =J_{0}\left(E_{1}\right) Y_{0}\left(E_{1}\right)+J_{1}\left(E_{1}\right) Y_{1}\left(E_{1}\right) .
\end{aligned}
$$

In the above equations, $J_{0}$ and $J_{1}$ are the Bessel functions of first kind of orders 0 and 1 , respectively. $Y_{0}$ and $Y_{1}$ are Bessel functions of second kind of orders 0 and 1, respectively. We must note that the results are properly adjusted. In addition, Figure 5 reveals that, for $\varepsilon_{h} \rightarrow 0$, the reflection coefficient $C_{R}$ for the lineal transitions, $n=1$, grows exponentially.

In Figure 6, we show the reflection coefficient $C_{R}$ and transmission coefficient $C_{T}$ as a function of the parameter $\kappa_{2}$ with constant values of $\varepsilon_{w}=1$ and $\varepsilon_{h}=0.25$.

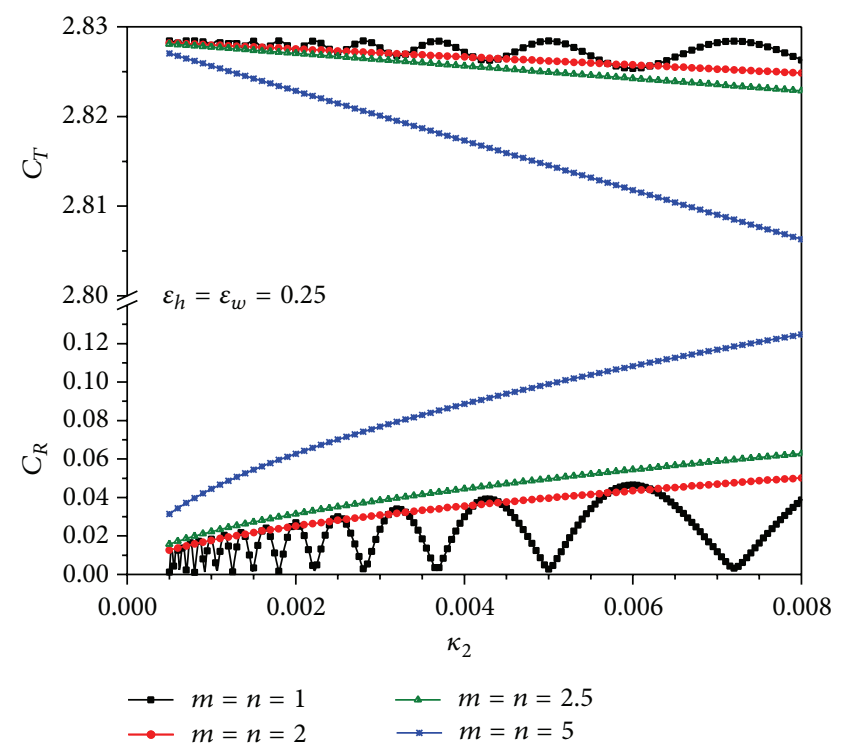

FIGURE 7: Reflection/transmission coefficients as a function of the kinematical parameter $\kappa_{2}$ with $\varepsilon_{h}=\varepsilon_{w}=0.25$ for four values of $m=n(=1,2,2.5,5)$.

The depth transitions are given for four values of $n=$ $(1,2,2.5,5)$. It should be noted that the reflection and transmission coefficients for a linear bottom transition have an oscillatory behavior with strong oscillations as $\kappa_{2} \rightarrow 0$. For those cases of $n>1$ the parabolic transition $(n=2)$ is less reflective than the other cases. The complementary transmission coefficients are shown at the top of the same figure. For values of the parameter $\kappa_{2} \rightarrow 0.008$, the bottom shape with $n=5$ has a transmission coefficient smaller than that of $n(=1,2,2.5)$. In the case of $\kappa_{2}=0.008$ the transmission coefficient for $n=5$ is $C_{T}=1.41297$ while that for $n=2$ is $C_{T}=1.41401$, which represents a difference of $7.4 \%$.

4.3. Wave Reflection by a Harbor with Variables Width and Depth. In Figure 7, we show the reflection and transmission coefficients for those harbors with variable geometry distribution with $m=n(=1,2,2.5,5)$ as a function of the parameter $\kappa_{2}$, with constant values of $\varepsilon_{h}=\varepsilon_{w}=0.25$. It is found that, for $\kappa_{2}=0.0005$, the maximum value of the transmission coefficient is near $C_{T}=2.827$ where always $C_{R} \rightarrow 0$, for the four harbor geometries. In the above limit, from (27) the maximum value of the transmission coefficient is $C_{T} \sim O\left(\varepsilon_{w}^{-1 / 2} \varepsilon_{h}^{-1 / 4}\right)$, which depends only on the width and depth ratios, $\varepsilon_{w}$ and $\varepsilon_{h}$, respectively. On the other hand, for a bigger value than $\kappa_{2}=0.0005$, that is, $\kappa_{2}=0.008$, the coefficient $C_{R}$ for $m=n=5$ takes a value of $C_{R}=0.125$ and the corresponding one for $m=n=2$ is $C_{R}=0.02$, which represents that a harbor with geometry distribution $m=n=5$ is 6.25 times greater than the case of $m=n=2$.

\section{Conclusions}

In this work, for the limit of $\kappa_{2} \ll 1$, we obtain simple asymptotic formulas for the reflection and transmission coefficients 
of linear long water waves, propagating in a tapered and slender harbor. The present mathematical formulation can be used as a first approximation to identify which harbors are less or more reflective than other geometries. The new formulas for the reflection and transmission coefficients are general and are very easy to apply.

The following summarizes the conclusions of the present work:

(1) It is found that a harbor with constant depth and variable width is more reflective than the case of harbors with constant width and variable depth.

(2) The results show that, for small values of the kinematic parameter $\kappa_{2}$, the harbors are less reflective and that the maximum value of the transmission coefficient is $C_{T} \sim O\left(\varepsilon_{h}^{-1 / 4} \varepsilon_{w}^{-1 / 2}\right)$, indicating that we can appreciate the motion of waves with large amplitudes for small values of $\varepsilon_{h}$ and $\varepsilon_{w}$.

(3) The combination of exponents $m \sim O(1)$ and $n \sim$ $O$ (1) can take fractional or integer values with $m \geq 1$ and $n \geq 1$.

(4) As an example, for a harbor with variables width and depth, $m=n=2$ and $\varepsilon_{w}=\varepsilon_{h}=0.25$, the results show that the energy flux balance, (27), $C_{R}^{2}+\varepsilon_{w} \varepsilon_{h}^{1 / 2} C_{T}^{2}=$ 0.999 is fully satisfied.

(5) The geometrical transitions studied in this work can be considered as idealized representations of harbors. The applications of the present formulas for the coefficients $C_{R}$ and $C_{T}$ are not limited to the studied cases in the present work, because the methodology used here can be extended to more complex harbor transitions.

\section{Conflict of Interests}

The authors declare that there is no conflict of interests regarding the publication of this paper.

\section{Acknowledgments}

This research is financially supported by the Research Grant no. Project SIP-20144038 from Instituto Politécnico Nacional. E. Bautista thanks the sabbatical program sponsored by Instituto Politécnico Nacional.

\section{References}

[1] Y. S. Li, S.-X. Liu, O. W. H. Wai, and Y.-X. Yu, "Wave concentration by a navigation channel," Applied Ocean Research, vol. 22, no. 4, pp. 199-213, 2000.

[2] R. L. Wiegel, Oceanographical Engineering, Dover Publications, 1992.

[3] A. B. Rabinovich, Handbook of Coastal and Ocean Engineering, World Scientific, Singapore, 2009.

[4] J. L. Luick and J. B. Hinwood, "Water levels in a dual-basin harbour in response to infragravity and edge waves," Progress in Oceanography, vol. 77, no. 4, pp. 367-375, 2008.
[5] M. Marcos, S. Monserrat, R. Medina, and P. Lomónaco, "Response of a harbor with two connected basins to incoming long waves," Applied Ocean Research, vol. 27, no. 4-5, pp. 209215, 2005.

[6] G. Bellotti, “Transient response of harbours to long waves under resonance conditions," Coastal Engineering, vol. 54, no. 9, pp. 680-693, 2007.

[7] C. C. Mei, M. Stiassnie, and D. K.-P. Yue, Theory and Applications of Ocean Surface Waves Part 1: Linear Aspects, vol. 23, World Scientific, 2005.

[8] B. Zanuttigh, S. M. Formentin, and R. Briganti, "A neural network for the prediction of wave reflection from coastal and harbor structures," Coastal Engineering, vol. 80, pp. 49-67, 2013.

[9] K. Kajiura, "On the partial reflection of water waves passing over a bottom of variable depth," in Proceedings of the Tsunami Meeting, vol. 24, pp. 206-230, University of Hawaii, AugustSeptember 1961.

[10] V. Rey, M. Belzons, and E. Guazzelli, "Propagation of surface gravity waves over a rectangular submerged bar," Journal of Fluid Mechanics, vol. 235, pp. 453-479, 1992.

[11] H. Lamb, Hydrodynamics, Cambridge University Press, Cambridge, UK, 6th edition, 1932.

[12] E. F. Bartholomeusz, "The reflection of long waves at a step," Mathematical Proceedings of the Cambridge Philosophical Society, vol. 54, pp. 106-118, 1958.

[13] D. G. Goring, Tsunamis-The Propagation of Long Waves onto a Shelf, California Institute of Technology, 1979.

[14] H.-K. Chang and J.-C. Liou, "Long wave reflection from submerged trapezoidal breakwaters," Ocean Engineering, vol. 34, no. 1, pp. 185-191, 2007.

[15] T.-H. Jung, K.-D. Suh, S. Oh Lee, and Y.-S. Cho, "Linear wave reflection by trench with various shapes," Ocean Engineering, vol. 35, no. 11-12, pp. 1226-1234, 2008.

[16] J.-J. Xie and H.-W. Liu, "An exact analytic solution to the modified mild-slope equation for waves propagating over a trench with various shapes," Ocean Engineering, vol. 50, pp. 7282, 2012.

[17] C. J. Bender and R. G. Dean, "Wave transformation by twodimensional bathymetric anomalies with sloped transitions," Coastal Engineering, vol. 50, no. 1-2, pp. 61-84, 2003.

[18] G. Wang, J. Zheng, Q. Liang, and Y. Zheng, "Analytical solutions for oscillations in a harbor with a hyperbolic-cosine squared bottom," Ocean Engineering, vol. 83, pp. 16-23, 2014.

[19] P. Lin and H.-W. Liu, "Analytical study of linear long-wave reflection by a two-dimensional obstacle of general trapezoidal shape," Journal of Engineering Mechanics, vol. 131, no. 8, pp. 822830, 2005.

[20] J.-J. Xie, H.-W. Liu, and P. Lin, "Analytical solution for long-wave reflection by a rectangular obstacle with two scour trenches," Journal of Engineering Mechanics, vol. 137, no. 12, pp. 919-930, 2011.

[21] H.-W. Liu, J.-X. Luo, P. Lin, and R. Liu, "Analytical solution for long-wave reflection by a general breakwater or trench with curvilinear slopes," Journal of Engineering Mechanics, vol. 139, no. 2, pp. 229-245, 2013.

[22] J. Harband, "Propagation of long waves over water of slowly varying depth," Journal of Engineering Mathematics, vol. 11, no. 2, pp. 97-119, 1977.

[23] E. G. Bautista, F. Méndez, O. Bautista, and A. Mora, "Propagation of shallow water waves in an open parabolic channel using the WKB perturbation technique," Applied Ocean Research, vol. 33, no. 3, pp. 186-192, 2011. 
[24] E. G. Bautista, J. M. Reyes, O. Bautista, and J. C. Arcos, "Deformation of water waves by geometric transitions with power law function distribution," World Academy of Science, Engineering and Technology, vol. 7, pp. 1416-1423, 2013.

[25] I. A. Svendsen, Introduction to Nearshore Hydrodynamics, vol. 24, World Scientific, 2006.

[26] W. M. Dingemans, Water Wave Propagation over Uneven Bottoms. Part 1-Linear Wave Propagation, vol. 1, World Scientific, 1997.

[27] R. G. Dean and R. A. Dalrymple, Water Wave Mechanics for Engineers and Scientists, vol. 2, World Scientific, 1984.

[28] J. Lighthill, Waves in Fluids, Cambridge Mathematical Library, 1978.

[29] R. G. Dean, "Long wave modification by linear transitions," Journal of the Waterways and Harbors Division, vol. 90, no. 1, pp. 1-30, 1964.

[30] J. Zwamborn and G. Grieve, "Wave attenuation and concentration associated with harbour approach channels," in Proceedings of the 14th International Conference on Coastal Engineering, pp. 2068-2085, ASCE, 1974.

[31] M. Rahman, Water Waves: Relating Modern Theory to Advanced Engineering Applications, vol. 3, Clarendon Press, New York, NY, USA, 1995. 


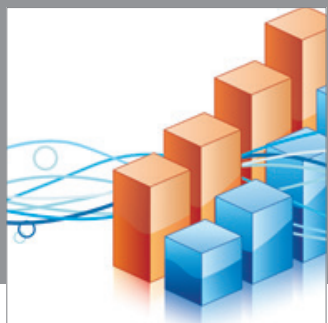

Advances in

Operations Research

mansans

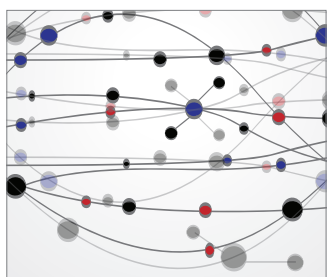

The Scientific World Journal
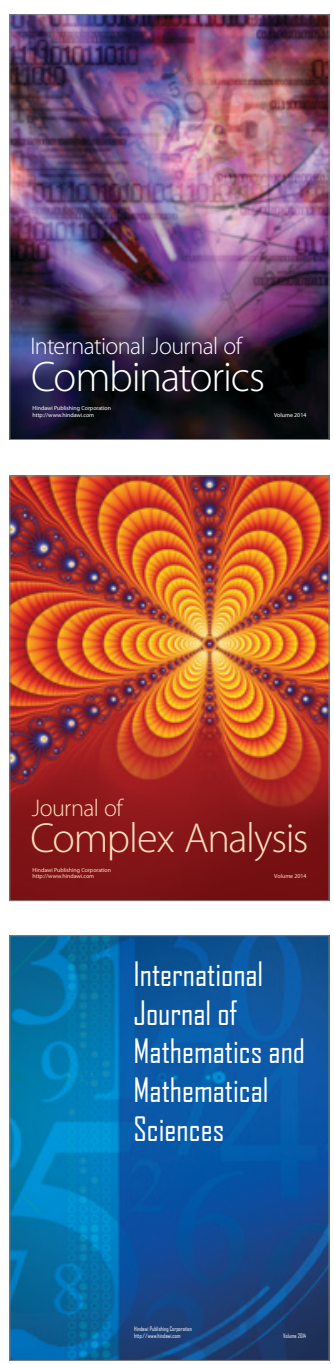
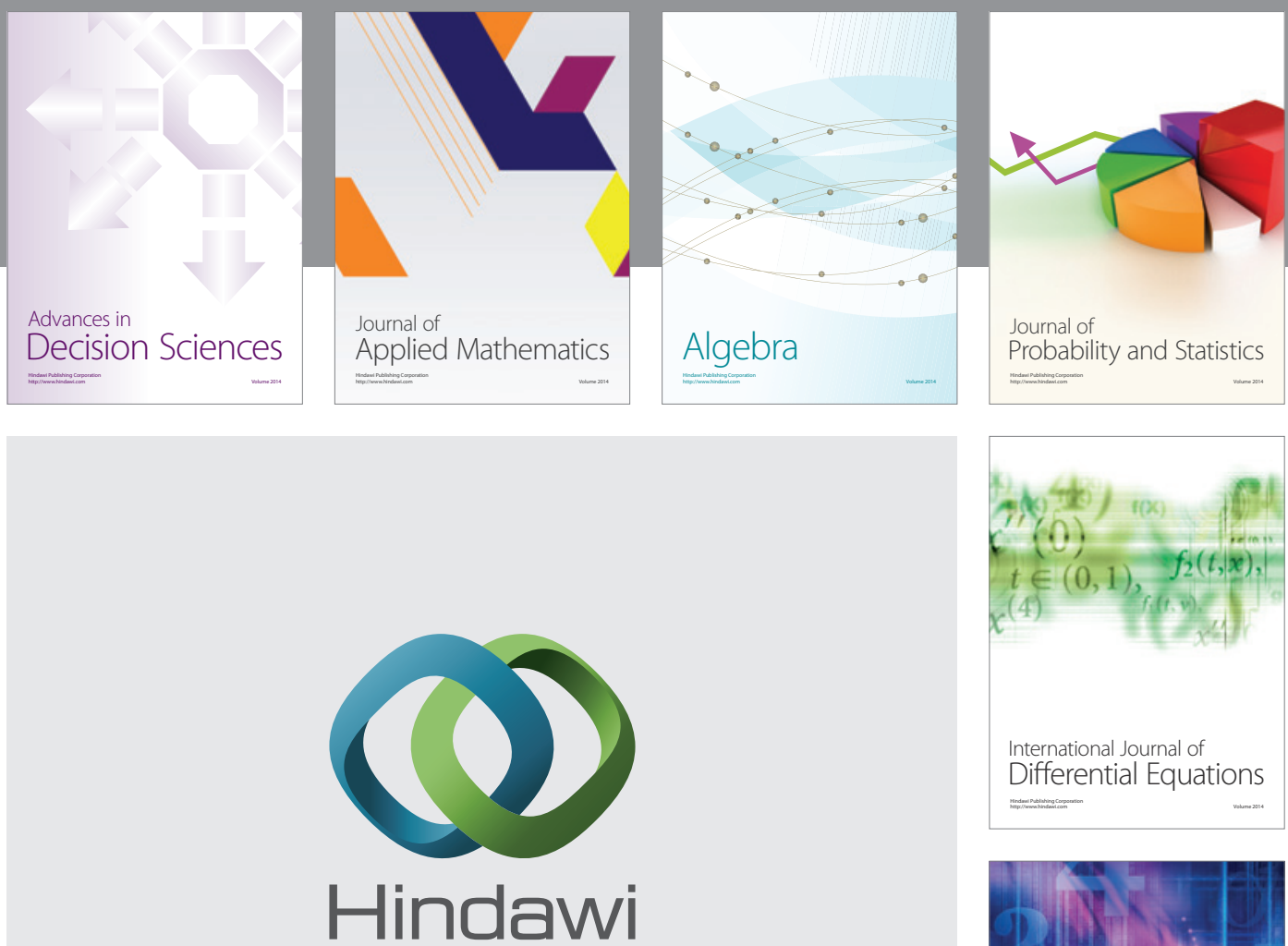

Submit your manuscripts at http://www.hindawi.com
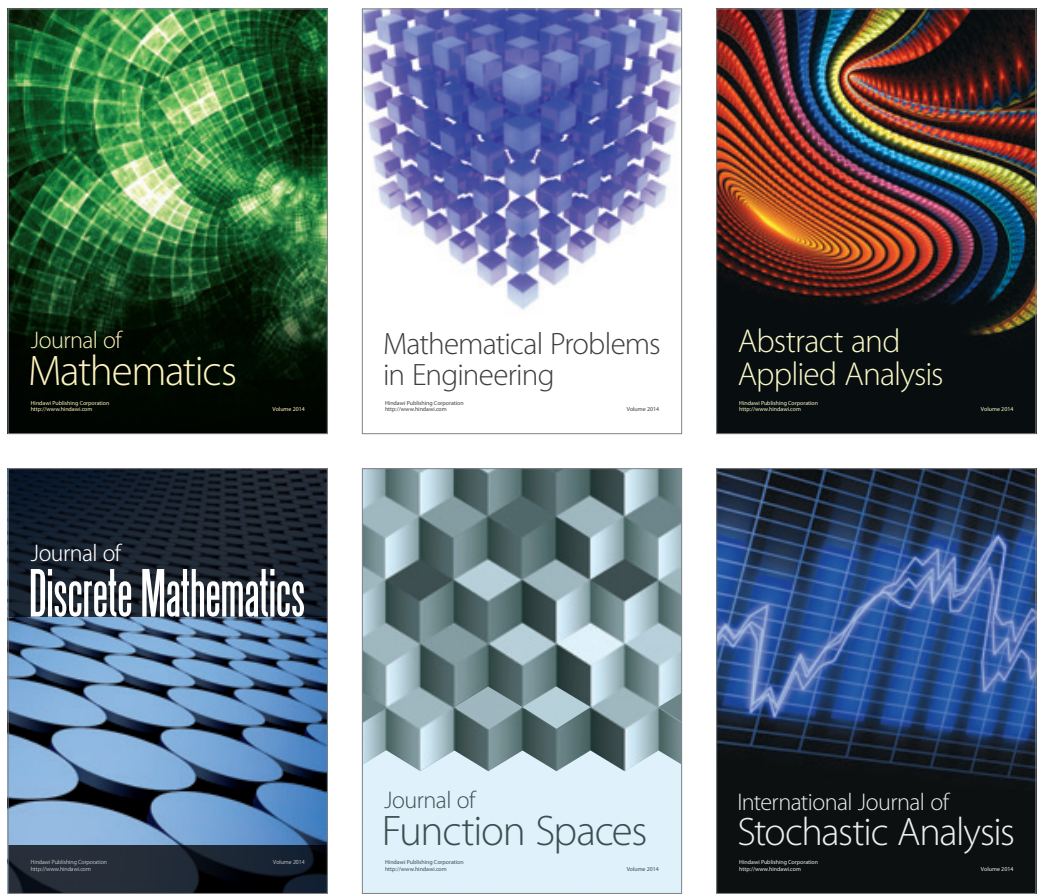

Journal of

Function Spaces

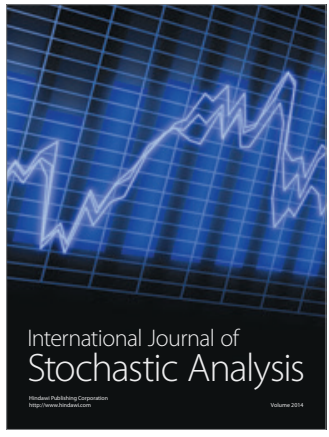

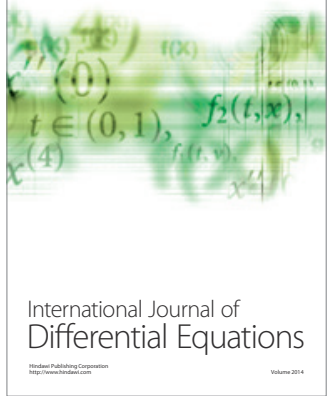
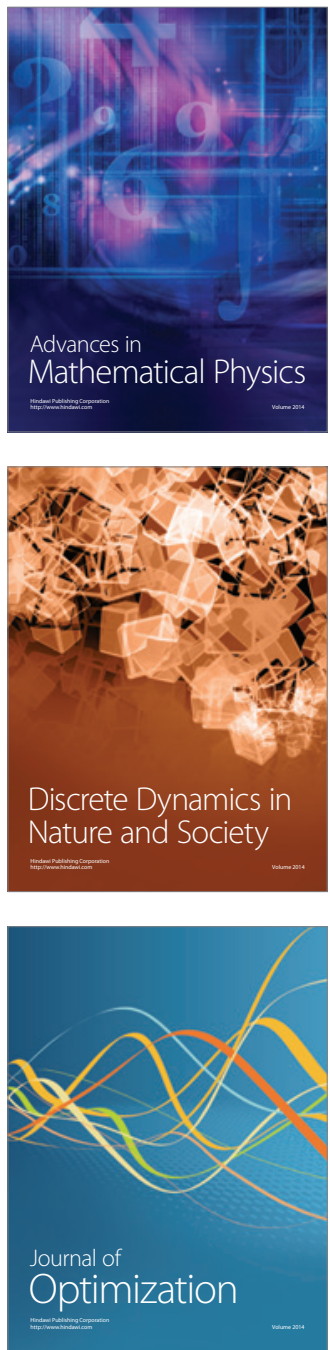